\title{
FEMTOSECOND RAMSEY FRINGES IN STRONGLY-DRIVEN RYDBERG SYSTEMS*
}

\author{
R.R. Jones \\ Physics Department, University of Virginia, Charlottesville, VA 22903 \\ C.S. Raman, D.W. Schumacher, and P.H. Bucksbaum \\ Physics Department, University of Michigan, Ann Arbor, MI 48109-1120
}

\begin{abstract}
The evolution of atomic systems under the influence of two coherent, temporally separated $100 \mathrm{fs}$ laser pulses has been studied. Ramsey interference fringes are observed in the populations of various energy levels as a function of delay between the two pulses. At high intensities the temporal interferograms are quite complicated and yield information on the couplings between all of the states in the atomic system. A simple three-level system as well as localized and dark radial Rydberg wavepackets have been studied.
\end{abstract}

\section{INTRODUCTION}

The experiments which will be described here were performed to study coherent excitation of several states by intense, short laser pulses. Such superposition states can be formed, for example, by utilizing the large coherent bandwidth in the laser pulse in single-photon excitation from the ground state to several higher lying levels. ${ }^{1}$ Alternatively, intensity-dependent energy shifts in the atom can allow the coherent excitation of many excited states whose energy spread far exceeds the bandwidth of the laser. ${ }^{2}$

Optical interferometers are often used to characterize the coherence of pulses of laser radiation. We have used an atomic bound-state interferometer to characterize bound superposition states in atoms. The interferometer utilizes two $100 \mathrm{fs}$ laser pulses in a time-domain optical double-resonance Ramsey technique, and is analogous to an optical interferometer. The first laser pulse coherently splits the initial bound-state of the atom into two or more final states. The states interact again during the second laser pulse. During the time between the two laser pulses all of the states aquire phase at different rates due to their different energies. The population in each state is modulated as a function of the delay between the first and second pulses.

Three experiments which study coherence in excited states of atoms were performed using the bound-state interferometer: (1) a simple three-level atom; (2) a radially localized Rydberg wavepacket; ${ }^{1}$ and (3) a "dark" or anti-wavepacket. ${ }^{3}$ Lowest order perturbation theory is found to be valid only at the lowest intensities studied, but the experimental results are adequately reproduced using a numerical integration of the time-dependent Schroedinger equation (TDSE) and a small Coulomb basis.

The short laser pulses are produced via chirped-pulse amplification of $100 \mathrm{fs}$ pulses from a self-mode locked Ti:Sapphire oscillator. Two spatially overlapped, 
temporally separated pulses are produced from a single pulse using a Michelson interferometer. The time delay between the two pulses is adjusted on a coarse scale by translating the retro-reflecting mirror in one leg of the interferometer. Fine adjustments $(\sim 0.02 \mathrm{fs})$ are achieved by rotating a glass slide in one arm of the interferometer, thereby increasing the optical path length in that arm.

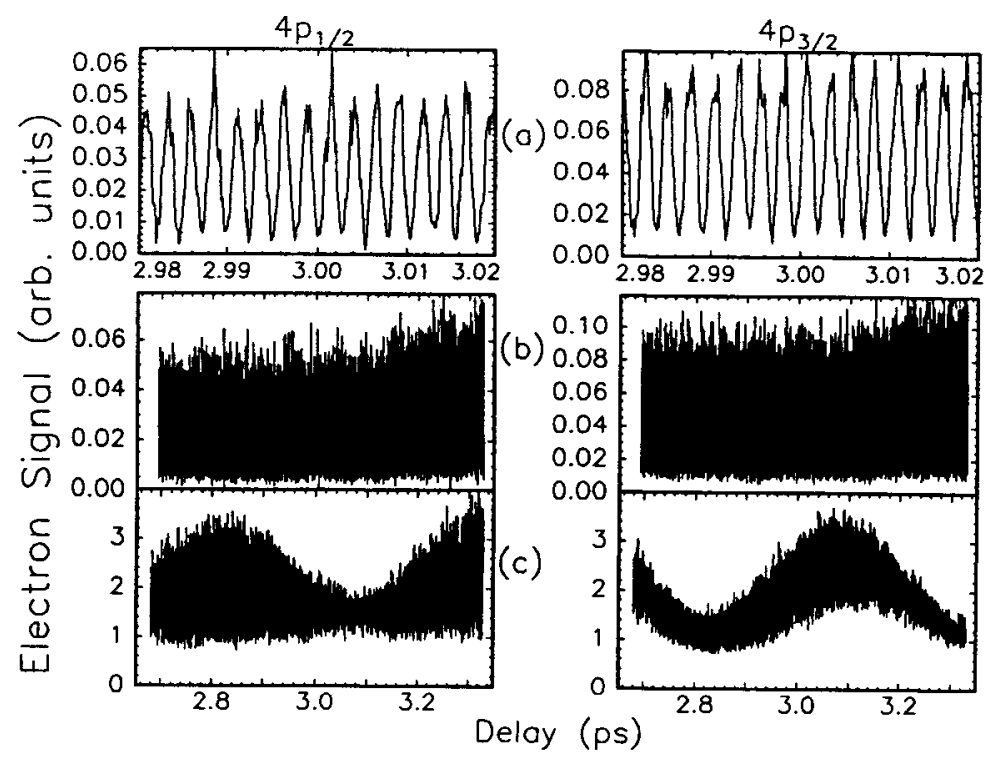

Figure 1. Ramsey interferograms showing population in both $4 \mathrm{p}$ fine structure states. (a) and (b) show the perturbative regime $\left(20 \mathrm{MW} / \mathrm{cm}^{2}\right)$ characterized by a sinusoidal modulation at the $4 s-4 p_{j}$ transition frequency. (c) shows the situation at higher intensity $\left(3 \mathrm{GW} / \mathrm{cm}^{2}\right.$ ) where an additional modulation at the fine-structure frequency appears due to ground state depletion and stimulated Raman effects.

\section{3-LEVEL SYSTEM}

In the first experiment the $100 \mathrm{fs}$ pulses coherently excite both fine-structure components of the $4 p$ resonance line from the $4 s$ ground state in a thermal beam of $K$ atoms. After the atoms have interacted with both short pulses a $5 \mathrm{~ns}$ dye laser interogates the population in both fine structure components of the $4 p$ state by exciting each level to a different nd Rydberg state. Population in the different Rydberg states is detected by using state selective field ionization.

Fig. 1 shows the population in both $4 p_{j}$ levels as a function of the delay between the two short laser pulses. The characteristic sinusoidal modulation (predicted by first order perturbation theory ${ }^{4}$ ) is clearly visible in the populations of both states in Fig. 1a. The population in each level oscillates with a characteristic frequency equal to its transition frequency from the ground state. The short pulses do not couple the $4 p$ states to each other, and therefore, the system can be described as two independent, two-level systems.

Fig. 1a is actually an expanded view of the interferograms shown in Fig. 1b. The 
results shown in Fig. 1c were obtained with a laser intensity 150 times greater than that in Fig. 1b. A slow modulation is evident in the interferograms for both 4p states with a characteristic period of $580 \mathrm{fs}$. The frequency of this oscillation matches the $4 \mathrm{p}$ fine structure splitting of $-57 \mathrm{~cm}^{-1}$. This additional interference is not predicted by lowest order perturbation theory and can be attributed to depletion of the ground state and stimulated Raman processes between the $4 p$ states via the $4 s$ level. The modulation is readily reproduced by a numerical integration of the full TDSE using the three relevant levels. Therefore, at high intensity the system can no longer be described as two independent two-level systems, and all levels are affected by the presence of the other two.

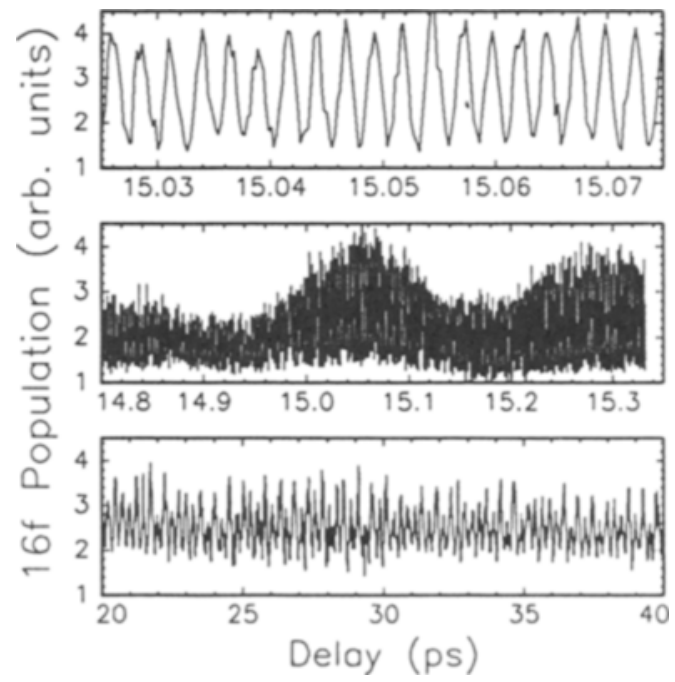

Figure 2. Interferogram of the $16 \mathrm{f}$ component of the radial wavepacket. All three plots were taken under identical conditions and are displayed with different time scales to show the modulations whose periods vary by nearly three orders of magnitude.

\section{LOCALIZED RADIAL WAVEPACKETS}

The second experiment was performed to study a similar but more complicated atomic system in which a radially localized wavepacket ${ }^{1}$ is formed by the coherent excitation of up to $10 \mathrm{nf}$ Rydberg states in $\mathrm{K}$. A $5 \mathrm{~ns}$ dye laser pulse transfers population from the ground state to the 3d state via two photon absorption. After a delay of $\sim 10 \mathrm{~ns}$, the atoms in the $3 \mathrm{~d}$ level are exposed to a $100 \mathrm{fs}, 772 \mathrm{~nm}$ laser pulse which coherently excites the nf $(12<\mathrm{n}<23)$ states. The wavepacket is then probed by an identical, second short pulse. The population in each Rydberg state is measured as a function of delay between the two short laser pulses using state selective field ionization. This type of differential measurement has not been possible in previous wavepacket studies which studied the evolution of the wavepacket using photoionization. ${ }^{1}$

Fig. 2 shows a typical interferogram of the population in the $16 \mathrm{f}$ state. All three scans were taken under identical conditions but are shown with different time scales 
to show the temporal modulations whose periods vary over nearly three orders of magnitude. At extremely low intensities, the $3 d$ state and nf Rydberg states can be characterized as many independent two level systems. However, at the laser intensity used to produce the spectra in Fig. 2, the sinusoidal oscillation predicted by first order perturbation theory ${ }^{4}$ is invalid. The discrete Fourier transform of these spectra show at least seven distinct oscillation frequencies, all of which correspond to difference frequencies between energy levels in the system. All of the different Rydberg states involved in the wavepacket exhibit unique temporal interferograms and Fourier transform spectra. Once again a numerical integration of the TDSE is used to reproduce the data. Good agreement between experiment and theory is achieved using only 22 basis states.

The complexity of the Ramsey interferograms for each Rydberg state indicates that the motion of the wavepacket is not the familiar motion of a classical particle which oscillates between the inner and outer turning points of its binding potential. Instead, the motion and degree of localization of the wavepacket can be quite complicated.

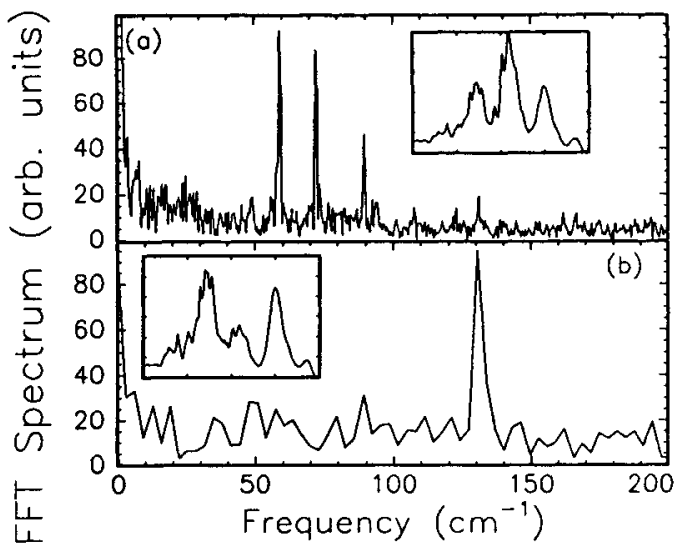

Figure 3. Discrete Fourier transform of the interferogram for the total Rydberg population for (a) a Gaussian frequency spectrum, and (b) a notched frequency spectrum. The broad features in the TOF electron signals in the insets correspond to individual Rydberg states (17f-13f) detected via state selective field ionization.

Because the bound-state interferometer allows us to determine the motion of the wavepacket so accurately, we have used the technique to study changes in the wavepacket motion caused by altering the frequency spectrum of the exciting laser pulse. Fig. 3a shows the frequency spectrum obtained when a wavepacket is excited with a $100 \mathrm{fs}$ Gaussian laser pulse. The inset in Fig. 3a shows the field ionization TOF spectrum which shows the relative amplitudes of the Rydberg states that are populated. The wavepacket oscillates with a central frequency of approximately 70 $\mathrm{cm}^{-1}$ which corresponds to the average spacing between the levels. The wavepacket is dispersive (i.e. its degree of localization changes with time) because the energy spacing between adjacent levels is not constant.

The spectrum shown in Fig. $3 \mathrm{~b}$ is obtained by placing a notch in the frequency 
spectrum of the short laser pulse which prevents excitation of the $15 \mathrm{f}$ state. The resulting TOF spectrum is shown again in the inset. Note that the wavepacket now oscillates primarily at a single frequency (i.e. without dispersion) and that the characteristic frequency of the wavepacket has been increased by a factor of two. (The 14f-16f energy spacing is now the dominate frequency difference). The loss of dispersion in the wavepacket is achieved at the expense of a decrease in localization (fewer states contribute to the wavepacket). More complicated schemes for "tailoring" wavepackets are also possible and can be easily studied using bound-state interferometry.

\section{"DARK" WAVEPACKETS}

Recently, Noordam et $\mathrm{al}^{3}$ showed that population in a single Rydberg level could be distributed over several Rydberg states if the initial state is exposed to a short, intense laser pulse. The redistribution can be seen in the time domain as the creation of a "hole" or "notch" in the Rydberg wavefunction which then moves through the otherwise stationary wavefunction in the same way as a localized wavepacket. ${ }^{3,5} \mathrm{We}$ have created such a "dark" wavepacket in K and have used the time-domain Ramsey technique to study the coherence and temporal evolution of the redistributed population.

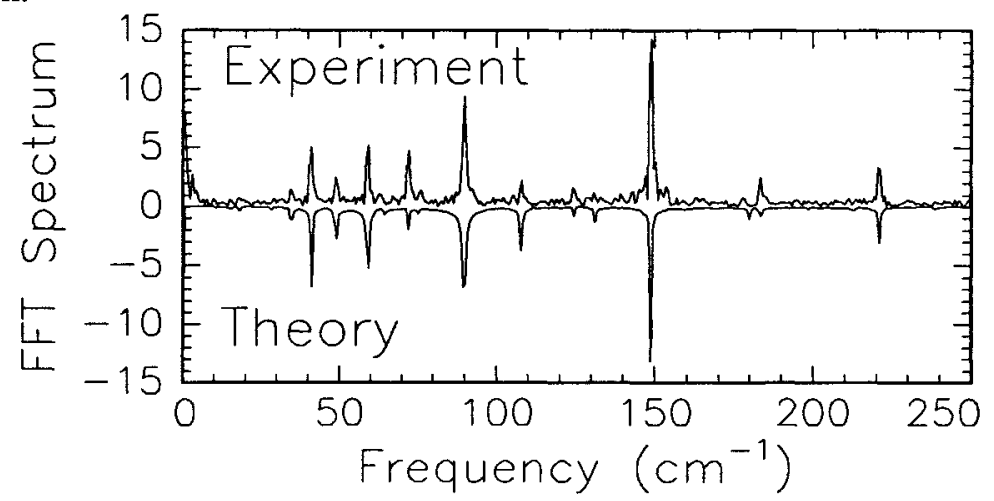

Figure 4. Discrete Fourier transform of the interferogram for the $18 \mathrm{f}$ population in a "dark" wavepacket created from an initial $15 f$ eigenstate at a laser intensity of $2 \times 10^{11} \mathrm{~W} / \mathrm{cm}^{2}$. The data is shown with positive amplitude and the theoretical prediction using 22 Coulomb basis states is shown with negative amplitude.

In the experiment, the $3 \mathrm{~d}$ state in $\mathrm{K}$ is populated via a two photon transition (as in the previous experiment) and a single nf Rydberg state is then populated by a second tunable dye laser. The Rydberg state is subjected to two short laser pulses approximately $300 \mathrm{~ns}$ after it is excited (This delay ensures that negligible population remains in the 3d level). The first pulse creates the "dark" wave packet by stimulating emission out of the Rydberg state to the $3 d$ state as well as absorption by the 3d state to different n'f Rydberg states. The second pulse then probes the system, either creating a second "hole" in the Rydberg wavefunction or filling in the first one. The redistribution is then probed using state selective field ionization.

Once again, multiple frequencies are apparent in the interferograms from a single 
Rydberg state indicating strong couplings between the Rydberg states via the $3 \mathrm{~d}$ level. As many as 20 different frequencies are observed in the Fourier transform of the time-domain spectrum from a single final state. Fig. 4 shows the Fourier transform of the $18 \mathrm{f}$ interferogram produced by redistribution from the $15 \mathrm{f}$ state. The experimental results are shown with positive amplitudes and the results of the 22 state numerical integration are shown with negative amplitudes. The agreement between theory and experiment is quite good.

The high resolution of this technique allows us to determine which Rydberg states contribute to the redistribution process. In this experiment only $f$ states play an important role in the formation of the dark wavepacket. Furthermore, we find that redistribution occurs to states which have binding energies which are either higher or lower than that of the initial state. Neither of these observations were possible using state-selective field ionization. ${ }^{4}$ Since the Fourier transform spectra show difference frequencies corresponding to all of the states which contribute to the wavepacket, we are able to identify all of the states in the superposition even if population in all of the levels is not directly detected.

\section{SUMMARY}

We have presented a time-domain, bound-state interferometric technique for studying coherence between excited states of atoms. The basics of the technique were demonstrated in the short-pulse excitation of a simple three-level system. Localized radial wavepackets in Rydberg states were also studied. The excellent signal-to-noise ratios in these experiments allowed a detailed study of the dynamics of these superposition states in the presence of strong laser fields. Furthermore, the technique has been used to characterize a "tailored" wavepacket created by altering the frequency spectrum of the short laser pulse. We have observed the time evolution of a "dark" wavepacket and the interferometric technique has allowed us to unambiguously determine which states contribute to the coherent superposition. The ability of the interferometric technique to identify states which are excited (but not directly detected) could contribute greatly to studies of coherent excitations in more complex systems.

*This work was performed at the University of Michigan.

\section{REFERENCES}

1. A. ten Wolde, L.D. Noordam, H.G. Muller, A. Lagendijk, and H.B. van Linden van den Heuvell, Phys. Rev. Lett. 61, 2099 (1988). J. A. Yeazell, M. Mallalieu, J. Parker, and C.R. Stroud, Jr., Phys. Rev. A 40, 5040 (1989). J.A. Yeazell and C.R. Stroud, Jr., ibid 43, 5153 (1991).

2. R.R. Freeman, P.H. Bucksbaum, H. Milchberg, S. Darack, D. Schumacher, and M.E.Geusic, Phys. Rev. Lett., 59, 1092 (1987). M.P. de Boer and H.G. Muller, ibid, 68, 2747 (1992). G.N. Gibson, R.R. Freeman and T.J. McIlrath, ibid, 69, 1904 (1992). R.R. Jones, D.W. Schumacher, and P.H. Bucksbaum, Phys. Rev. A, 47, R49 (1993). M.P. de Boer, L.D. Noordam, and H.G. Muller, ibid 47, R45 (1993).

3. L.D. Noordam, H. Stapelfeldt, D.I. Duncan, and T.F. Gallager, Phys. Rev. Lett. 68, 1496 (1992).

4. L.D. Noordam, D.I. Duncan, and T.F. Gallager, Phys. Rev. A. 45, 4734 (1992).

5. R.R. Jones and P.H. Bucksbaum, Phys. Rev. Lett. 67, 3215 (1991). H. Stapelfeldt, D.G. Papaioannou, L.D. Noordam, and T.F. Gallagher, ibid 67, 3223 (1991). 